\title{
factores responsables de la corrosión de las armaduras embebidas en el hormigón
}

CESAR DEL OLMO RODRIGUEZ, Dr. en Ciencias Químicas del IETcc

\section{sinopsis}

$628-4$

\begin{abstract}
El presente trabajo pasa revista a las características del hormigón que sirven de protección de las armaduras embebidas en este material; en el trabajo se incluyen unas cifras que pueden servir de pauta para valorar estas características.
\end{abstract}

\section{INTRODUCCION}

El hormigón y el acero, como es sabido, constituyen dos materiales perfectamente compatibles, con similares coeficientes de dilatación térmica, cuyas características se complementan. La unión de ambos materiales, que se conoce con el nombre de hormigón armado o pretensado $\left({ }^{*}\right)$, participa de la excelente resistencia mecánica a la compresión del hormigón, a lo que se suma una adecuada resistencia a la flexión que el acero aporta y de la que el hormigón carece.

La durabilidad del hormigón armado depende, como es lógico, de la durabilidad de los dos materiales que lo componen. Ambos materiales se comportan de forma distinta ante la acción del medio exterior, siendo el acero el más sensible de ambos materiales al efecto de dicho medio, y por lo tanto, el menos durable de los dos.

(*) Esta diferencia entre estos dos tipos de hormigón estriba en el procedimiento de fabricación, siendo además distintas, en uno y otro caso, la sección de las armaduras, de menor diámetro en los hormigones pretensados.
El acero puede ser atacado (corroído) por la humedad ambiente y por el oxígeno del aire, a cuyo efecto se suma el de otros agentes nocivos, tales como el anhídrido sulfuroso $\left(\mathrm{SO}_{2}\right)$, que con frecuencia contamina las atmósferas urbana e industrial, y los cloruros existentes en el ambiente costero.

Pero el hormigón, en general, es capaz de proteger suficientemente a las varillas y alambres de acero, que constituyen las armaduras, del medio exterior agresivo antes señalado, formando el conjunto -hormigón armado - un material de durabilidad aceptable.

\section{PROTECCION DEL ACERO POR EL HORMIGON}

La capacidad protectora del hormigón hacia las armaduras reside en una triple característica de este material.

En primer lugar, el hormigón interpone entre las armaduras y el medio exterior una barrera física eficaz, capaz de reducir, hasta valores despreciables en la práctica, el acceso hasta el acero de los agentes agresivos arriba citados.

Además, el hormigón tiene la propiedad de conferir a las armaduras rodeadas por él un estado de pasivación (o estado en el que es imposible la corrosión), originado por la alta alcalinidad que este material genera. Dicho estado se produce como consecuencia de la formación, en la superficie de las armaduras, de una capa protectora alcalina constituida por los productos de la reacción entre el metal y el hormigón.

$\mathrm{Y}$, por último, el hormigón presenta una baja conductividad eléctrica, lo que hace que, después de endurecido, quede poco favorecido el paso de corriente eléctrica $(0$, lo que es igual, la transferencia iónica) a través de su masa; paso de corriente que de adquirir una cierta magnitud también puede ser responsable de la corrosión (o pérdida de materia) de las armaduras. 


\subsection{Porosidad del hormigón $y$ espesor del recubrimiento de las armaduras}

La barrera protectora de hormigón no es eficaz cuando este material tiene una porosidad alta $\mathrm{y} / \mathrm{o}$ el espesor del recubrimiento de hormigón de las armaduras no es el adecuado en función del grado de agresividad que presenta el medio que rodea al hormigón armado.

Como datos de referencia para juzgar la porosidad de un hormigón, cabe indicar que la porosidad máxima teórica de este material es del $9 \%$; la porosidad de un hormigón de buena calidad se cifra entre el 13 y el $20 \%$, siendo la porosidad de un hormigón de baja calidad superior al $25 \%$ (1).

Por su parte, pueden tomarse los valores contenidos en el cuadro I como punto de referencia para estimar la eficacia del recubrimiento del mortero u hormigón de las armaduras en relación con el medio donde se encuentra ubicado este material (2).

Una alta porosidad en el hormigón puede ser debida a una baja calidad de este material, a la aparición de fisuras profundas en su superficie $y / o$ ser consecuencia de un ataque sufrido por el hormigón.

Entre los factores que, integrantes en la calidad del hormigón, influyen en el aumento de la porosidad de este material, cabe citar los siguientes: el empleo en el hormigón de una curva incorrecta de sus áridos (discontinua, carente de la proporción adecuada de finos, etc.] ; la utilización de una proporción inadecuada de agua de amasado (muy alta o demasiado baja), o de una baja dosificación de cemento; la mala compactación del hormigón, etc.
Por su parte, la aparición de ísuras profundas en el hormigón puede ser consecuencia de defectos habidos: en los materiales que forman parte del hormigón láridos reactivos, cementos expansivos, o de elevado calor de hidratación, etc.); en la fabricación del hormigón (p. e., curado deficiente, que ha generado retracciones en su masa); en el proyecto (hormigón menos dimensionado o de inferiores resistencias que las necesarias) y/o en el uso del hormigón (por estar sometido a sobrecargas no previstas en el proyecto).

El ataque al hormigón por los agentes que le son nocivos (sulfatos, aguas muy puras, etcétera) afecta sobre todo a la pasta cementante, que forma el entramado de unión entre los áridos, produciendo la consecuente disgregación de estos áridos y el correspondiente aumento de la porosidad del hormigón. En casos como los citados, por los huecos que en el hormigón origina este ataque, penetra con facilidad hasta las armaduras los agentes agresivos mencionados. Estos agentes, al actuar sobre las armaduras, producen un efecto multiplicador de destrucción del hormigón, por cuanto en el interior de su masa se crean tensiones mecánicas debidas al aumento de volumen que supone la formación de herrumbre en la superficie de las armaduras, cuyo volumen es muy superior al del acero primitivo.

Cabe apuntar, por último, que el tratamiento térmico del hormigón puede alterar las características protectoras del revestimiento de hormigón de las armaduras, disminuyendo su eficacia. 
Valores orientativos del espesor mínimo que debe tener el recubrimiento de mortero u hormigón de las armaduras para que su protección sea eficaz.

\begin{tabular}{|c|c|c|c|}
\hline $\begin{array}{c}\text { Distancias mínimas a las paredes del encofrado o a la superficie lisa } \\
\text { del hormigón }\end{array}$ & $\begin{array}{c}\text { Valor minimo } \\
\text { absoluto }\end{array}$ & $\begin{array}{l}\text { Valor referido al } \\
\text { diámetro del contorno } \\
\text { aparente de la barra } \\
\text { más gruesa }\end{array}$ & $\begin{array}{l}\text { Valor referido a la } \\
\text { dimensión máxima } \\
\text { del árido }\end{array}$ \\
\hline $\begin{array}{l}\text { Elementos de obras interiores, en un ambiente estable y } \\
\text { no corrosivo. }\end{array}$ & $1 \mathrm{~cm}$ & $1 \mathrm{vez}$ & $1 \mathrm{vez}$ \\
\hline $\begin{array}{l}\text { Elementos de obras exteriores protegidos contra la lluvia } \\
\text { o la helada en el estado de saturación. } \\
\text { Obras enterradas en un suelo no corrosivo. }\end{array}$ & $2 \mathrm{~cm}$ & 2 veces & $1 \mathrm{vez}$ \\
\hline $\begin{array}{l}\text { Elementos de obras interiores expuestos a influencias } \\
\text { atmosféricas intensas (tales como fuertes precipitaciones, } \\
\text { alternancias de humedad y de sequedad pronunciadas en } \\
\text { estado de saturación). } \\
\text { Elementos expuestos a heladas intensas y a la acción de } \\
\text { sal para el deshielo. }\end{array}$ & $3 \mathrm{~cm}$ & 1,25 veces & 1,5 veces \\
\hline $\begin{array}{l}\text { Elementos de obras que deben presentar una resistencia } \\
\text { particular bien a los agentes químicos, bien a la abrasión. }\end{array}$ & \multicolumn{3}{|c|}{$\begin{array}{l}\text { La distancia mínima debe ser fijada por el Ingeniero- } \\
\text { Proyectista. }\end{array}$} \\
\hline
\end{tabular}

\subsection{Alcalinidad}

El hormigón, tal como se ha señalado anteriormente, presenta una elevada alcalinidad, que constituye una protección importante para las armaduras recubiertas por este material. Esta alcalinidad puede ser parcialmente neutralizada por la acción del anhídrido carbónico del aire, fenómeno que se conoce con el nombre de carbonatación del hormigón y que, con frecuencia, sólo afecta a una capa superficial del material de pequeño espesor (2 a $5 \mathrm{~mm}$ ), sin que dicha carbonatación, en general, llegue a penetrar hasta la masa de hormigón próxima a las armaduras.
Sin embargo, la profundidad de la carbonatación del hormigón y el consiguiente efecto sobre las armaduras, depende a su vez de la porosidad del hormigón, del espesor de recubrimiento de hormigón, del medio donde se encuentre ubicado y de la edad del material; esta carbonatación puede ser particularmente rápida en hormigones ligeros y celulares y en los morteros de las viguetas cerámicas donde van embebidos los alambres en ese tipo de elementos resistentes.

El efecto de la carbonatación puede ser importante cuando el hormigón armado está situado en un ambiente muy contaminado, como puede ser un medio industrial donde el hormigón está afectado por humos y gases; o en un medio urbano muy polucionado; o también en el caso del hormigón colocado en un garaje mal ventilado. En casos como los citados, la carbonatación puede afectar peligrosamente a las armaduras si el hormigón no posee una compacidad suficiente y el espesor del recubrimiento de hormi- gón de las armaduras no es el idóneo.

El valor de la alcalinidad del hormigón también disminuye por el empleo de cementos con adiciones $\left({ }^{*}\right)$ como conglomerantes del hormigón armado. En tales casos, el valor del potencial de hidrógeno $(\mathrm{pH})$ del hormigón es menor que el que alcanza con un cemento portland $y$, por lo tanto, resulta inferior la capacidad de pasivación de las armaduras que este tipo de hormigones posee.

(*) El cemento portland, al hidratarse, desprende hidróxido cálcico, que es el componente responsable de la alcalinidad del hormigón. En los cementos con adiciones, al contener, por una parte, menor proporción de clínker de portland y por otra, al combinarse el hidróxido cálcico formado con las adiciones activas (puzolanas, escorias o cenizas volantes) contenidas en estos cementos, la alcalinidad potencial o reserva alcalina de estos cementos es menor que la del portland. 


\subsection{Conductividad}

En estudios experimentales de laboratorio (3) se ha encontrado que para un mismo medio existe mayor riesgo de corrosión de armaduras en hormigones fabricados con cenizas volantes, en relación con el riesgo que existe para los mismos hormigones, pero preparados con cemento portland; aunque este hecho no ha sido puesto de manifiesto en la práctica, posiblemente porque la mayor capacidad de los hormigones con cenizas volantes para conseguir una menor porosidad, compensa el efecto que la disminución de la alcalinidad comporta.

Por último, produce asimismo una disminución de la alcalinidad del hormigón, con el riesgo antes señalado, el ataque o lixiviación del hormigón por deslavado con aguas muy puras. Este ataque origina la pérdida del cemento, que es precisamente el componente que al hidratarse produce la alcalinidad que el hormigón desarrolla.

Se ha puesto de manifiesto que la corrosión de los alambres se inicia a partir de que el pH del hormigón, cuyo valor normal para un cemento portland es del orden de 12 , desciende por debajo de 9,5. Este límite, que tiene un valor orientativo, queda modificado cuando en el hormigón concurren otros factores, como son la existencia de una concentración importante de cloruros, cuyo efecto se estudia en el apartado siguiente; dicha presencia de cloruros dificulta o impide la formación de la capa protectora de pasivación que, en ausencia de este ion, normalmente se crea alrededor de las armaduras, tal como se ha hecho referencia en el apartado 2 de este trabajo.
El hormigón es un material poco conductor de la electricidad, cu y a conductividad, además, disminuye a medida que su edad aumenta, como consecuencia de que el paso del tiempo va produciendo, por una parte, el secado del hormigón $y$, por otra, la colmatación de los poros de este material y, consecuentemente, la reducción de las posibilidades del paso de humedad por el interior de su masa.

Sin embargo, la conductividad eléctrica del hormigón aumenta notablemente cuando este material contiene determinados componentes (o iones) que actúan como electrolitos (es decir, como conductores). Entre estos iones cabe destacar en primer lugar los cloruros, aunque también presentan esta propiedad otros constituyentes del hormigón, como son los sulfatos y nitratos, este último de presencia menos frecuente en el hormigón.

El factor conductividad tiene, en relación con el riesgo de corrosión de las armaduras que nos ocupa, una importancia relativa superior que la de los otros dos factores considerados (porosidad y alcalinidad del hormigón), porque, de hecho, la presencia en el hormigón de una concentración apreciable de los iones citados, responsables a su vez de dicho aumento de conductividad, es una de las causas más frecuentes de la corrosión de las armaduras del hormigón.

La presencia en el hormigón de estos iones tiene su origen bien en sus componentes (cemento, áridos, agua de amasado y/o aditivos), o bien en la contaminación exterior del hormigón, posterior a su fabricación.
Como es suficientemente conocido el efecto perjudicial que sobre las armaduras del hormigón ocasionan estos iones, todas las normas e instrucciones que rigen el proyecto y la ejecución de obras de hormigón armado y pretensado fijan limitaciones para el contenido de estos iones en los componentes del hormigón, aunque sería más adecuado fijar limitaciones al contenido total de dichos iones en el hormigón, en lugar de establecerlo en los componentes por separado.

A título de ejemplo, en el cuadro II se incluyen los contenidos máximos de los dos iones agresivos más frecuentes -cloruros y sulfatos-, admitidos en algunos de los componentes del hormigón por varias de las normas e instrucciones europeas vigentes. 
CUADRO II

Limitaciones establecidas por algunas normas europeas a los contenidos de cloruros y sulfatos en los componentes del hormigón.

\begin{tabular}{|c|c|c|c|c|c|c|c|c|}
\hline \multirow{2}{*}{ PA I S } & \multirow{2}{*}{$\begin{array}{c}\text { NORMA } \\
0 \\
\text { INSTRUCCION }\end{array}$} & \multirow{2}{*}{$\begin{array}{c}\text { TIPO } \\
\text { DE } \\
\text { HORMIGON }\end{array}$} & \multicolumn{3}{|c|}{$\begin{array}{l}\text { CONTENIDO MAXIMO DE } \mathrm{Cl}^{-}[\%] \\
\text { PERMITIDO EN: }\end{array}$} & \multicolumn{3}{|c|}{$\begin{array}{c}\text { CONTENIDO MAXIMO DE SO }{ }_{3}(\%) \\
\text { PERMITIDO EN: }\end{array}$} \\
\hline & & & Arena & Grava & $\begin{array}{l}\text { Agua de } \\
\text { amasado }\end{array}$ & Arena & Grava & $\begin{array}{l}\text { Agua de } \\
\text { amasado }\end{array}$ \\
\hline \multirow{2}{*}{ España } & $\mathrm{EH}-73$ & Armado & - & - & 0,6 & 1,0 & 1,0 & 0,083 \\
\hline & EP-77 & Pretensado & 0,03 & 0,03 & 0,025 & 0,41 & 0,1 & 0,083 \\
\hline \multirow{2}{*}{ Inglaterra } & The greater & Armado & - & - & - & - & - & - \\
\hline & Council & Pretensado & 0,06 & 0,018 & - & - & - & - \\
\hline \multirow{2}{*}{ Holanda } & \multirow{2}{*}{ NEN 3542} & Armado & 0,1 & 0,05 & - & - & - & 一 \\
\hline & & Pretensado & 0,01 & 0,005 & - & - & - & 一 \\
\hline \multirow{2}{*}{ Alemania Federal } & \multirow{2}{*}{ DIN 4226} & Armado & - & - & - & - & - & - \\
\hline & & Pretensado & 0,02 & 0,02 & - & - & - & 一 \\
\hline \multirow{2}{*}{ Alemania del Este } & & Armado & - & - & 0,1 & - & - & - \\
\hline & & Pretensado & 一 & - & 0,05 & - & - & 一 \\
\hline \multirow{2}{*}{ Austria } & \multirow{2}{*}{$\begin{array}{c}\text { ÖNORM } \\
\text { B3304 }\end{array}$} & Armado & - & - & - & - & - & 一 \\
\hline & & Pretensado & 0,01 & 0,01 & - & 0,15 & 0,15 & - \\
\hline
\end{tabular}

Por lo que respecta al empleo de aditivos aceleradores del fraguado y del endurecimiento del hormigón, preparados con cloruro, que constituye el origen más frecuente de este constituyente en el hormigón, la vigente Instrucción española $\mathrm{EH}-73$, que es la que más directamente nos afecta, sólo hace referencia al empleo del cloruro cálcico, que es el aditivo más conocido y de uso más frecuente para el fin indicado. Sin embargo, dicha Instrucción no se pronuncia sobre la proporción máxima en que este aditivo puede ser usado en los hormigones armados, aunque indirectamente establece como límite máximo el valor de 1,5 a $2 \%$ del peso del cemento, que fija para los hormigones en masa; asimismo, prohibe el uso del cloruro cálcico "cuando son de temer acciones de carácter electroquímico", aunque "puede admitirse su empleo si se justifica previamente que no supone peligro alguno, presente ni futuro, para las armaduras", lo que siempre resulta difícil de justificar. El empleo de este aditivo queda totalmente prohibido en los hormigones pretensados.

Otras normas europeas establecen prescripciones en relación con el ion cloro, no para los constituyentes del hormigón considerados aisladamente, sino para el material en su conjunto. Así, en el DTU (4) se establece que el contenido total de cloruros ("), expresados como ion $\mathrm{Cl}^{-}$, deberá ser en los hormigones armados como máximo del $0,65 \%$ del peso del cemento. En esta línea se define la norma italiana de tuberías de hormigón armado y pretensado (5), que admite para el hormigón un contenido máximo total de iones cloro solubles en agua del 0,02 por 100 del peso del cemento.

${ }^{*}$ ) Determinado como suma del contenido de $\mathrm{Cl}$ - en los áridos, agua, cemento y aditivos.
Por su parte, ciertos autores (6) señalan que el nivel admisible de cloruros en el hormigón no debe considerarse aisladamente, sino en relación con el $\mathrm{pH}$ del material, de tal manera que en un hormigón con un valor de $\mathrm{pH}$ inferior al normal, el efecto de los cloruros sobre las armaduras se acentúa; más aún, se ha encontrado (7) que en presencia de una proporción alta de cloruros puede producirse en las grietas que se originan en los aceros de alta resistencia una disminución puntual o muy localizada de $\mathrm{pH}$, independiente del $\mathrm{pH}$ del electrolito (hormigón en nuestro caso), que favorece la corrosión del acero; disminución de $\mathrm{pH}$ que es manifestación de alguna forma de fragilización por hidrógeno del acero. 


\section{MEDIO AGRESIVO}

Como se ha hecho referencia en párrafos anteriores, los tres factores que determinan la capacidad protectora de un hormigón hacia sus armaduras están condicionados por las características del medio donde está situado el hormigón.

El contenido de humedad del medio exterior es la característica más importante del mismo en relación con la durabilidad del acero embebido en un hormigón, siguiendo en importancia el contenido de iones agresivos (principalmente, cloruros) presentes en dicho medio (*).

(*) Se prescinde aquí de la consideración de los agentes agresivos al propio hormigón (aguas y terrenos sulfatados, aguas muy puras, hielo, etc.), que se sale del tema del presente trabajo, aunque su importancia en la durabilidad de los hormigones armados y pretensados es obvia.
El efecto combinado de los factores citados está presente en los hormigones armados y pretensados sumergidos en agua de mar, donde se producen deterioros frecuentes; aunque la experiencia muestra que puede transcurrir un largo tiempo antes de que aparezcan estos problemas de corrosión. Cuando se manifiestan estos daños, las zonas del hormigón más afectadas son las que se encuentran sometidas a ciclos alternativos de humedad y secado, localizadas principalmente en las zonas de salpicaduras.

\section{BIBLIOGRAFIA}

1. Publicación Técrica del CERILH, núm. 144, 28/30 de enero de 1963.

2. Propuesta de recomendaciones internacionales para el proyecto y la ejecución de obras de hormigón CEB-FIP, mayo 1970.

3. Influence des cendres volantes sur la protection des armadures dans le béton. "Cahiers du Centre Scientifique et Technique du Bâtiment", núm. 165.

4. Documento Técnico Unificado (DTU), núm. 1.467, de octubre de 1977.

5. Proposta di Norme relative alle tubazioni in cemento armato precompreso per acquedotti ed irrigazione. "L'Industria Italiana del Cemento", febrero 1972.

6. Corrosion of reinforcement in concrete. Rilem, núm. 51, mayojunio 1976.

7. B. F. BROWN: 4th Int. Cong. on Met. Corr., Extended Abstracts, p. 18, Amsterdam, septiembre 1969.

CALLEJA, J.: “Corrosión de armaduras en los cementos armados y pretensados". Informes de la Construcción, núm. 180, mayo 1966.

"Instrucción para el proyecto y la ejecución de obras de hormigón en masa o armado », EH-73 (Decreto 3062/1973, de 19-X-73). "Instrucción para el proyecto $y$ la ejecución de obras de hormigón pretensado", EP-77 (Real Decreto 1408/1977, de 18-II-77). "Pliego de prescripciones técnicas generales para la recepción de cementos", RC-75 (Decreto 1964/1975, de 23-V-75).

\section{résumé}

Facteurs responsables de la corrosion des armatures noyées dans le béton

César del Olmo Rodríguez, Dr. ès Sciences chimiques

Dans cet article, l'auteur passe en revue les caractéristiques du béton qui servent de protection des armatures noyées dans ce matériau. II donne des chiffres qui peuvent servir de

\section{summary}

Factors responsible for rust in concrete-encased reinforcements

César del Olmo Rodríguez, Dr. of Chemical Sciences

The present article sums up the characteristics of the concrete which protects the reinforcements imbedded in this material This study includes some figures which can be used as a guide for evaluating these characteristics.

\section{zusammenfassung}

Für die Korrosion der in Betan eingelassenen Bewehrungen verantwortliche Faktoren

César del Olmo Rodríguez, Dr. der Chemischen Wissenschaften

Die vorliegende Arbeit zeigt die Merkmale Die vorliegende Arbeit zeigt die Merkmale
des Betons auf, welche zum Schutz der in
dieses Material eingelassenen Bewehrumgen dieses Material eingelassenen Bewehrungen dienen; die Arbeit beinhaltet verschiedenen Ziffern, welche als Ausgangspunkt für die 